\title{
Pyoderma Gangrenosum in the Urologist Clinic
}

\author{
Dina J. Ludwig ${ }^{a} \quad$ Hossain Roshani ${ }^{a} \quad$ Martijn G. Steffens ${ }^{b} \quad$ Frederik C. Moll $^{c}$ \\ Robertus G. Teepe ${ }^{d}$
}

aDepartment of Urology and d Dermatology, Haga Teaching Hospital, The Hague; bepartment of Urology and 'Pathology, Isala

Clinics, Zwolle, Netherlands

\section{Key Words}

Penis • Pyoderma gangrenosum $\cdot$ Scrotum $•$ Ulcer $•$ Urology

\begin{abstract}
Pyoderma gangrenosum is a rare non-infectious skin disorder. It is often associated with systemic diseases, like the inflammatory bowel disease, rheumatological disease and (hematological) malignancy. The diagnosis is affirmed through a process of elimination and is principally based on clinical presentation and course. We present a 59-year-old male with T-cell large granular lymphocyte leukemia and pyoderma gangrenosum of penis and scrotum. Finally the patient was successfully treated with systemic prednisolone.
\end{abstract}

Copyright $\odot 2015$ S. Karger AG, Basel

\section{Introduction}

Genital ulcers can be classified as infectious or non -infectious disorders. Infectious causes usually concern sexually transmitted diseases. Banal ulcers are also seen (especially caused by an infection with Streptococci

\section{KARGER}

Fax +4161306 1234

E-Mail karger@karger.ch

www.karger.com
(C) 2015 S. Karger AG, Basel

1015-9770/15/0093-0159\$38.00/0

Accessible online at:

www.karger.com/cur spp.). Non-infectious causes are usually related to a malignancy, inflammatory bowel disease, rheumatological disease, Behcet's disease or trauma. It is also favorable to consider more exceptional diagnoses as leukocytoclastic vasculitis or pyoderma gangrenosum (PG) in some cases $[1,2]$.

\section{Case Report}

A 59-year-old male was referred to the urology out-patient clinic with an expanding ulcer on his penis and scrotum. He was recently diagnosed with leukemia (T-cell large granular lymphocyte leukemia) and was treated by cyclophosphamide and pegfilgrastim (recombinant human granulocyte colony stimulating factor). The ulcer on the penis and scrotum was one centimeter in diameter. It was a red, granulating tissue with jagged wound edges. There was edema of the prepuce. No other skin abnormalities were seen. There was no lymphadenopathy in the groin. Cultures of the ulcer showed a Klebsiella pneumonia and a proteus mirabilis. Biopsies of the ulcer gave no indication for a malignancy (no localization of T-cell large granular lymphocyte leukemia in particular). The additional research was inconclusive. Laboratory test did not show an increased CRP or leukocyte level. Serology for herpes simplex virus, Chlamydia trachomatis and Treponema pallidum were negative.

Treatment with imipenem, cilastatin, clindamycin and fluconazole was started serially in collaboration with the medical microbiologist. In the course of 10 days, the ulcer increased in size and reached dimensions of $3 \times 5 \mathrm{~cm}$. Given the above, it was 

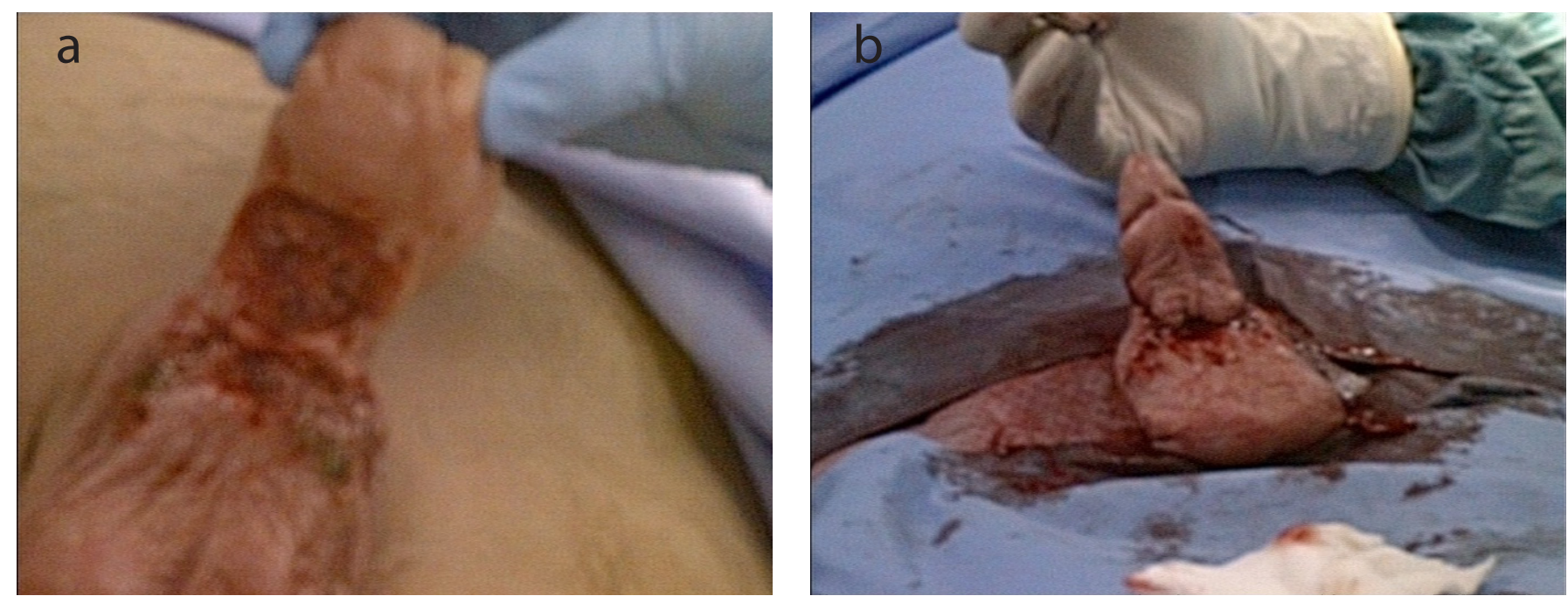

Fig. 1. Ulcus per-operative: before (a) and after (b) resection and reconstruction.

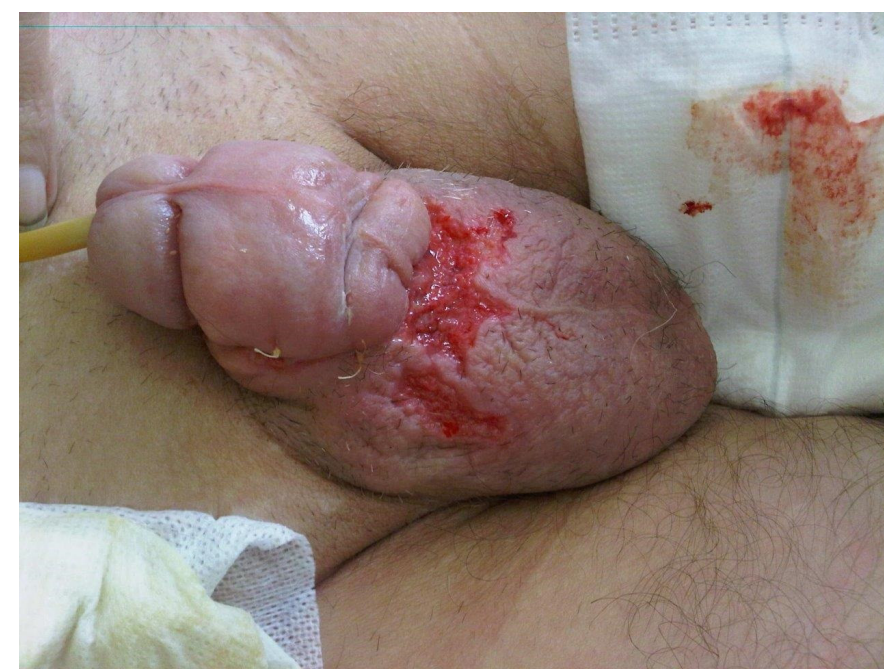

Fig. 2. Ulcus one week post-operative.

thought to be an insufficient healing ulcer by edema and decubitus. It was decided to surgically remove the ulcer and reconstruct the wound to promote healing (fig. 1). During the operation new cultures and biopsies were taken. Wound healing started initially well but ceased after 3 days (fig. 2). The extra cultures yield no clues, and particularly no Haemophilus ducreyi, Calymmatobacterium granulomatis or Streptococci spp. were found. The biopsies showed an active non-specific inflammation (neutrophil inflammatory infiltrate). No malignancy was found. Two small new ulcers with crustae on both knees were observed a week later. There was not a clear cause for those wounds. Two weeks later, one of the wounds on the knees expanded and had an ulcer with a whimsical, dark colored edge formed. It was decided to ask the dermatologist in consultation. The dermatologist immediately diagnosed PG, based on the typical raised edge of the ulcer on the knee. Also the neutrophilic inflammatory infiltrate seen in the biopsy of the knee and in retrospect in the biopsy of the prepuce fitted this diagnosis (fig. 3). The patient was subsequently treated with prednisolone $40 \mathrm{mg}$ oral for 4 weeks. Ulcers of all sites healed within 4 weeks. The dose of prednisolone was then lowered with $5 \mathrm{mg}$ a week.

\section{Discussion}

PG is a rare non-infectious neutrophilic dermatosis with poorly understood pathogenesis, commonly unknown to urologists. The incidence is estimated to be $3-10$ per $1,000,000$ per year [3, 4]. It is characterized by (chronic recurrent) painful ulcerations of the skin. Localization usually involves the lower extremities, but they can also occur on the face, in the neck, on the scrotum and penis [3]. PG has been rarely described on the penis and the scrotum [1-5]. Diagnosing PG is not always easy and is primarily based on the clinical presentation and its clinical course. It presents as an inflammatory papule or hemorrhagic pustule rapidly evolving into an ulcer. The most notable characteristic of PG is an ulcer with undermined dark colored borders and a necrotic and mucopurulent base. PG causes no lymphadenopathy or lymphadenitis. $\mathrm{PG}$ is associated with underlying systemic diseases in $50 \%$ of cases, such as inflammatory bowel 


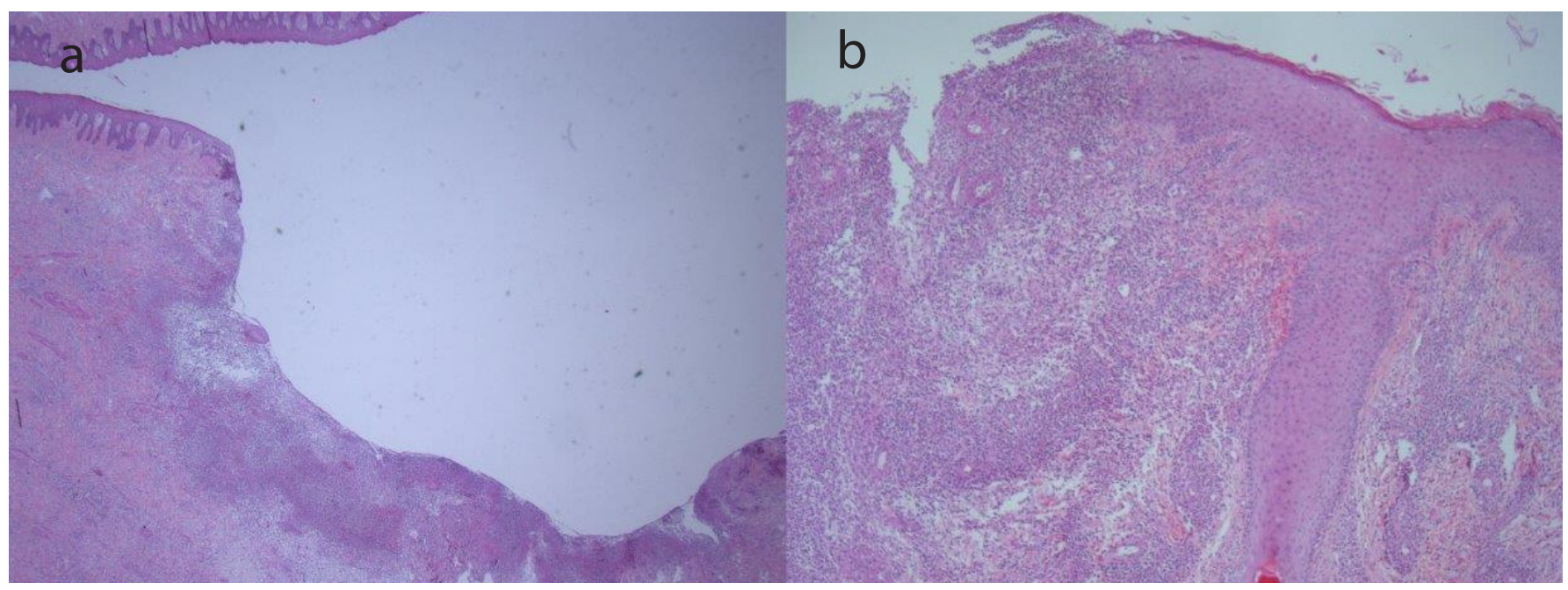

Fig. 3. Deep ulcus in biopsy prepuce (a) and biopsy knee (b).

disease, rheumatoid arthritis and (hematological) malignancies $[1,2]$. Our patient initially missed the typically dark inflamed wound edges at genital sites but developed that typical sign later on the knees, which led to the right diagnosis. PG should be considered for non-healing ulcers on penis and/or scrotum and when other specific causes are excluded. Furthermore, poor healing after resection, especially with a non-specific pathological rash with neutrophil inflammatory infiltrate is a reason to consider this diagnosis [1-3]. The so-called Köbner phenomenon is observed in $20 \%$ of cases. Köbner phenomenon describes the recurrent character of the lesion after a previously non-specific lesion of the skin or a surgical intervention [3]. This is also observed to be the case in the described patient. In general, the histopathological finding in PG is similar to a non-specific ulceration with abscess formation. It is centered in the dermis. The adjacent dermis shows acute and chronic inflammation with vasculitis-like changes. Hemorrhage is often present and eosinophils may be evident (fig. 3). The diagnosis is primarily established by exclusion. It cannot be overemphasized how important accurate clinical information is in establishing the correct diagnosis [6]. PG is often treated with immunosuppressive drugs such as local or systemic corticosteroids [5]. Other therapeutic modalities include azathioprine, cyclosporine, cyclophosphamide, colchicine, dapsone, clofazimine, mercaptopurine, minocycline, mycophenolate mofetil, sulfasalazine, TNF- $\alpha$-thalidomide, tracolimus and blockers (Adalimumab and Inflixi- mab) [2]. Pegfilgrastim is sometimes used as a treatment for PG, but restraint is provided, since it also can initiate an exacerbation of PG $[3,4]$. This might be possible in this case. Prednisolone orally was started on which the patient soon had a good response and healed ulcers.

PG is a rare, non-infectious and painful ulcerative disease, belonging to the spectrum of neutrophilic dermatoses. It is important to consider this diagnosis in penile and scrotal ulcers, especially in patients with a systemic disease or malignancy, with an atypical clinical presentation and course of the ulcer. Urologist should be aware of the diagnosis.

\section{Informed Consent}

Informed consent is provided by the patient to publish anonymously. 


\section{References}

1 Lee DK, Hinshaw M, Cripps D, Jarrard DF: Pyoderma gangrenosum of penis. J Urol 2003;170:185-186.

2 Badgwell C, Rosen T: Penile pyoderma gangrenosum. Dermatol Online J 2006;12:8.
3 Ruocco E, Sangiuliano S, Gravina AG, Miranda A, Nicoletti G: Pyoderma gangrenosum: an updated review. J Eur Acad Dermatol Venereol 2009;23:1008-1017.

4 Trémezaygues L, Schmaltz R, Vogt T, Reichrath J: Management des pyoderma gangrenosum. An update on clinical features, diagnosis and therapy. Hautarzt 2010;61:345-355.
5 Kim TH, Oh SY, Myung SC: Pyoderma gangrenosum of the penis. J Korean Med Sci 2009;24:1200-1202.

6 Patterson JW: Weedon's Skin Pathology, 4th Edition. Elsevier, 2015.

\section{Erratum}

Unfortunately, an author name 'Omer G. Doluoglu' was written as 'Ömer Doluoğlu' by mistake in the paper by Ceylan et al.: Can the free/total PSA ratio predict the gleason score before prostate biopsy?. Curr Urol 2015;9:24-27. 